\title{
EFEITO DO MANEJO DE PLANTAS DANINHAS NO DESENVOLVIMENTO INICIAL DE Pinus taeda EM VÁRZEAS NA ARGENTINA ${ }^{1}$
}

\author{
Edison Bisognin Cantarelli ${ }^{2}$, Sérgio Luiz de Oliveira Machado ${ }^{3}$, Ervandil Corrêa Costa ${ }^{3}$ e Raul Pezzutti ${ }^{4}$
}

\begin{abstract}
RESUMO - Realizou-se um estudo sobre o efeito de cobertura e períodos de manejo de plantas daninhas em plantios no ano de 1999 de Pinus taeda, localizados na Província de Corrientes, Argentina. Em razão das características da área, várzeas, foram construídos camalhões de $1,80 \mathrm{~m}$ de largura por $0,60 \mathrm{~m}$ de altura para o plantio das mudas e, a seguir, instaladas parcelas com três fileiras de 12 plantas em cada uma no espaçamento de 1,75 m entre as mudas e 4,0 m entre o centro dos camalhões. Foram medidas somente as 10 plantas do camalhão central, com o objetivo de avaliar o efeito de diferentes modalidades e intensidades de manejo de plantas daninhas na sobrevivência (\%), no desenvolvimento inicial em altura $(\mathrm{cm})$, no diâmetro do colo $(\mathrm{cm})$ e no fator de produtividade $\left(\mathrm{cm}^{3}\right)$ das mudas de Pinus taeda. Avaliaram-se as modalidades de controle: controle químico na linha do plantio (camalhão) e controle químico em área total sendo avaliados por dois períodos: um ano e dois anos de controle, tendo ainda uma testemunha, sem nenhum controle. O delineamento estatístico do experimento foi em blocos ao acaso, com três repetições. Diferenças significativas foram obtidas entre os tratamentos de controle químico em relação ao sem controle. Os resultados levaram à conclusão de que é benéfico o controle por dois períodos e que não houve diferença quanto às modalidades de controle (camalhão e área total). As mudas de Pinus taeda foram submetidas ao teste de Tukey para analise da sobrevivência e não apresentaram diferença significativa a $5 \%$ de probabilidade de erro nas médias.
\end{abstract}

Palavras-chave: Silvicultura, proteção florestal e herbicidas.

\section{EFFECT OF WEED MANAGEMENT ON THE INITIAL DEVELOPMENT OF Pinus taeda IN LOW FLATLANDS OF ARGENTINA}

\begin{abstract}
A study on the effect of vegetation cover and weed management periods on plantings of Pinus taeda was carried out in the Province of Corrientes, Argentina, in 1999. Due to the area characteristics, low flatlands, $1.80 \mathrm{~m}$ wide $\times 0.60 \mathrm{~m}$ high ridges were built for seedling planting. Following, plots with 3 rows of 12 plants each, at the spacing of $1.75 \mathrm{~m}$ between plants and $4 \mathrm{~m}$ between the center of the ridges, were installed. Only 10 plants in the central ridge were measured in order to evaluate the different methods and intensity of weed management in survival rate $(\%)$, initial height development $(\mathrm{cm})$, stem diameter $(\mathrm{cm})$ and seedling yield $\left(\mathrm{cm}^{3}\right)$ of Pinus taeda. The following control methods were evaluated: chemical control in the planting area (ridge) and chemical control in the whole area were evaluated twice: one and two years of chemical control, with control treatment and without control treatment. The experimental design was completely randomized with three repetitions. Significant differences were found between the chemical control treatments compared to the treatment without control. The results showed that the chemical control during 2 periods is beneficial, and no differences between control methods (ridge and total area) were found. Survival data of Pinus taeda seedlings were examined by the Tukey's test and showed no significant differences between the means at $5 \%$ probability.
\end{abstract}

Keywords: Silviculture, forest protection, herbicides.

\footnotetext{
${ }^{1}$ Recebido em 27.03.2003 e aceito para publicação em 05.04.2006.

${ }^{2}$ Departamento de Engenharia Rural da Universidade Federal de Santa Maria -UFSM. E-mail: <engedison@ yahoo.com>.

${ }^{3}$ Departamento de Defesa Fitossanitária da UFSM, prédio 42.97105-900 Santa Maria-RS.

${ }^{4}$ Departamento de Suporte Técnico da Empresa Bosques del Plata S.A. Posadas-Argentina.
} 


\section{INTRODUÇÃO}

O setor florestal, com as múltiplas funções das florestas, traz consigo numerosos benefícios fundamentais para o homem, como o desenvolvimento social e econômico, a redução da pobreza, a geração de empregos, a energia rural, o abastecimento de produtos florestais essenciais, a conservação do solo e da água, a recuperação de áreas degradadas, entre outros. Estudos da Organização das Nações Unidas para a Agricultura e a Alimentação (FAO) estimam que no ano de 2010 haverá um déficit mundial de cerca de 700 milhões de $\mathrm{m}^{3}$ de madeira roliça (SAGPyA FORESTAL, 1997).

O aumento de produtividade será somente possível com o controle dos fatores limitantes do crescimento e desenvolvimento das árvores. Dentro desses fatores se destacam os que estão relacionados com a presença de plantas daninhas, pois estas competem por água, luz, elementos nutritivos e espaço, podendo ainda, exercer pressão de natureza alelopática, atuando como hospedeiros intermediários de pragas e doenças, prejudicando a colheita e outras operações silviculturais e aumentando o risco de incêndios (WIECHETECK, 1988; citado por KOGAN e FIGUEROA, 1998). Segundo Pitelli e Marchi (1988), a interferência imposta pelas plantas daninhas às culturas florestais é mais severa, principalmente, na fase inicial de crescimento, ou seja, do transplante até cerca de 1 ano de idade.

Na Argentina, áreas de várzeas estão sendo muito utilizadas para o plantio de florestas, uma vez que possuem menor valor comercial e se distribuem em grandes extensões nas áreas em que as empresas do setor florestal estão instaladas. Mais de $80 \%$ dos 6.000 ha plantados pela empresa Bosques del Plata S.A., em 1999, foram realizados em solos de várzeas na forma de camalhões. As empresas localizadas no nordeste argentino têm um gasto elevado no controle de plantas daninhas, chegando a constituir $27 \%$ dos custos de implantação da floresta. Qualquer redução nos custos do controle terá grande incidência na rentabilidade das empresas florestais dessa região.

De acordo com Montenegro (1998), o controle de plantas daninhas em fileira, assim como o controle total, tem apresentado resultados significativos em relação a testemunhas sem controle, em áreas com relevo alto.
Dessa forma, é importante avaliar o desenvolvimento inicial de Pinus taeda, bem como sua sobrevivência sob influência de uma flora daninha típica desse ambiente.

Nesse contexto, os aspectos anteriormente apresentados motivaram a busca de respostas de plantios de Pinus taeda diante de um manejo de plantas daninhas, tendo como objetivos:

a)Definir a melhor modalidade e intensidade de manejo de plantas daninhas em plantios de Pinus taeda em solos de várzeas.

b) Avaliar a resposta das plantas de Pinus taeda diante das diferentes modalidades e intensidades de manejo de plantas daninhas.

\section{MATERIAL E MÉTODOS}

O experimento foi conduzido em solo de várzea dentro da Unidade Cartográfica $\mathrm{n}^{\circ}$ 38, que apresenta a seguinte associação: Kandihumultes típicos (Diaz de Vivar): Argiloso Vermelho-Amarelo Distrófico; Kandiudalfes sódicos ( $\mathrm{A}^{\circ}$ Itaimbé): Argiloso Vermelho Eutrófico Sódico; e Distrocreptes líticos (Sosa Cué): Neossolo Litólico Distrófico.

O solo enquadra-se melhor dentro da série Sosa Cué, que apresenta características de relevo normal, posição "média loma" e "média loma baja". A topografia do local é suave, com ondulações mais ou menos elevadas, declividade de 1 a $2 \%$, guardando entre si pouca diferença de altitude relativa (MONTENEGRO, 1999).

O clima da região, segundo a Classificação Climática de Köppen, pertence ao tipo Cfa, ou seja, clima subtropical úmido. A temperatura média anual é de 19,1 ${ }^{\circ} \mathrm{C}$, com temperatura média do mês mais frio (julho) de $12,3{ }^{\circ} \mathrm{C}$ e a média do mês mais quente (janeiro) de $25,3^{\circ} \mathrm{C}$. Essas temperaturas próprias de verão e inverno relativamente elevadas, e sua pequena variação anual, definem a esse clima como sendo mesotérmico. A precipitação da região é do tipo isoidro (precipitação igualmente distribuída nos 12 meses do ano), com valor médio anual de $1.680 \mathrm{~mm}$, destacando-se como mais chuvosos dois períodos, um no outono (abril e maio) e outro na primavera (setembro e outubro). Em 1999, os valores médios de chuva se mantiveram entre um mínimo normal de $21 \mathrm{~mm}$ em janeiro e um máximo de $301 \mathrm{~mm}$ em maio, conforme Tabela 1 . 
Efeito do manejo de plantas daninhas no desenvolvimento ...

Tabela 1 - Valores médios mensais de temperatura e precipitação na Estação Meteorológica de Santo Tomé, 1999 Table 1 - Mean monthly temperature and rainfall at Santo Tomé Meteorological Station, 1999

\begin{tabular}{|c|c|c|c|c|c|c|}
\hline \multirow[t]{2}{*}{ Mês } & \multicolumn{3}{|c|}{ Temperatura $\left({ }^{\circ} \mathrm{C}\right)$} & \multirow{2}{*}{$\begin{array}{c}\text { Precipitação } \\
\text { Dia/mês }\end{array}$} & \multirow{2}{*}{$\begin{array}{c}\text { Precipitação } \\
(\mathrm{mm})\end{array}$} & \multirow{2}{*}{$\begin{array}{c}\text { Precipitação } \\
\text { Acumulada }\end{array}$} \\
\hline & MIN. & MAX. & MED. & & & \\
\hline$\overline{\text { Jan }}$ & 18,5 & 32,2 & 25,3 & 04 & 21,0 & 28,0 \\
\hline Fev & 18,2 & 30,4 & 24,3 & 03 & 164,0 & 192,0 \\
\hline Mar & 18,6 & 30,7 & 24,6 & 05 & 50,0 & 242,0 \\
\hline Abr & 13,1 & 22,7 & 17,9 & 11 & 214,0 & 456,0 \\
\hline Mai & 9,9 & 19,4 & 14,6 & 05 & 301,0 & 757,0 \\
\hline Jun & 8,6 & 17,2 & 12,8 & 06 & 95,0 & 852,0 \\
\hline Jul & 7,8 & 16,8 & 12,3 & 10 & 76,0 & 928,0 \\
\hline Ago & 9,2 & 20,8 & 14,9 & 02 & 28,0 & 949,0 \\
\hline Set & 11,6 & 22,9 & 17,3 & 08 & 197,0 & 1146,0 \\
\hline Out & 12,5 & 23,7 & 18,1 & 10 & 137,0 & 1283,0 \\
\hline Nov & 14,0 & 27,5 & 20,7 & 04 & 79,0 & 1362,0 \\
\hline$\underline{\text { Dez }}$ & 18,0 & 32,4 & 25,2 & 08 & 100,0 & 1462,0 \\
\hline MIN & 7,8 & 16,8 & 12,3 & - & 21,0 & - \\
\hline MAX & 18,6 & 32,4 & 25,3 & - & 301,0 & 一- \\
\hline MED & 13,2 & 24,6 & 18,8 & 6,3 & 161,0 & - \\
\hline
\end{tabular}

A amostragem do solo para a análise química e física foi realizada em $1^{\circ}$ de setembro de 2000 e processada no Laboratório de Ecologia Florestal do Departamento de Ciências Florestais da Universidade Federal de Santa Maria, seguindo-se a metodologia adotada por Tedesco et al. (1995) (Tabela 2).

O experimento foi instalado entre 25 e 28 de agosto de 1999. Ao redor da área experimental foram construídos canais de drenagem com a finalidade de remover o excesso de água da área.

Em pré-transplante das mudas (abril de 1999), a área experimental foi dessecada através da aplicação aérea da mistura de glyphosate (Roundup) a $1,44 \mathrm{~kg}$ $\mathrm{ha}^{-1}\left(3,0 \mathrm{~L} \mathrm{ha}^{-1}\right)$ com atrazine (Agar Cross Atrazina) a 1,50 $\mathrm{kg} \mathrm{ha}^{-1}\left(3,0 \mathrm{~L} \mathrm{ha}^{-1}\right)$ acrescidos do adjuvante (Dowfax) na concentração de $0,5 \% \mathrm{v} / \mathrm{v}$.

Após a dessecação das plantas daninhas, construíram-se camalhões de 1,80 m (largura) x 0,60 m (altura), onde foram transplantadas as mudas de Pinus taeda. Para a confecção dos camalhões, espaçados 4,0 m um do outro, foi utilizado um entaipador marca "Rastra Savana" tracionada por trator com potência de 300 HP. Na oportunidade, o solo foi adubado com $200 \mathrm{~kg} \mathrm{ha}^{-1}$ de superfosfato triplo. Após a abertura das covas no camalhão, o transplante das mudas foi realizado manualmente numa distância de $1,75 \mathrm{~m}$ uma da outra, totalizando 36 mudas por parcela $\left(252 \mathrm{~m}^{2}=\right.$ 21 x 12 m), divididas em três camalhões.

Tabela 2 - Resultados das análises química e física de uma amostra do solo da área experimental, retirada em 2000. Santa Maria, $\mathrm{RS}^{1}$

Table 2 - Chemical and physical analysis of soil sample from the experimental area, collected in 2000. Santa Maria, $R S^{I}$

\begin{tabular}{|c|c|c|c|}
\hline \multirow[t]{2}{*}{ Determinações } & \multirow[t]{2}{*}{ Análise Química ${ }^{2}$} & \multicolumn{2}{|l|}{ Análise Física } \\
\hline & & Distribuição do tamanho das partículas & $\%$ \\
\hline$\overline{\mathrm{pH}\left(\mathrm{H}_{2} \mathrm{O} 1: 1\right)}$ & 4,8 & Argila (< que $0,002 \mathrm{~mm})$ & 27,0 \\
\hline $\mathrm{pH}$ & 5,9 & & \\
\hline $\mathrm{P}(\mathrm{ppm})$ & 3,2 & & \\
\hline $\mathrm{K}(\mathrm{ppm})$ & 25,0 & & \\
\hline Al $\left(\mathrm{cmol} \mathrm{L}^{-1}\right)$ & 0,9 & Classe Textural ${ }^{3}-3$ & \\
\hline $\mathrm{Ca}\left(\mathrm{cmol} \mathrm{L}{ }^{-1}\right)$ & 5,5 & Franco-arenoso & \\
\hline $\operatorname{Mg}\left(\mathrm{cmol} \mathrm{L}^{-1}\right)$ & 1,4 & & \\
\hline M.O. $(\%)$ & 2,61 & & \\
\hline
\end{tabular}

${ }^{1}$ Laboratório de Ecologia Florestal do Departamento de Ciências Florestais da Universidade Federal de Santa Maria. ${ }^{2}$ Tedesco et al. (1995).

${ }^{3}$ Vettori (1969). 
As mudas utilizadas no experimento foram produzidas no viveiro da própria empresa. O sistema de produção foi em tubetes dispostos em bandejas com 40 cavidades cada uma. As mudas foram transplantadas quando apresentavam em média 3,0 mm de diâmetro do colo.

Para a aspersão dos herbicidas foi utilizado pulverizador costal pressurizado com ar comprimido contendo pontas Lechler AD 120-015, operando a 2 bares de pressão e com vazão correspondente a 200 $\mathrm{L} \mathrm{ha}^{-1}$ de calda.

O delineamento experimental foi de fatorial $(2 \times 2+1)$ disposto em blocos ao acaso, com três repetições. $\mathrm{O}$ tratamento adicional foi representado pela testemunha. Foi determinado como área útil da parcela, o camalhão central entre as 10 plantas centrais da parcela, evitando, assim, o efeito de tratamentos vizinhos.

O experimento foi instalado com as fileiras orientadas no sentido norte-sul. De acordo com Silva (1998), a orientação norte-sul é a mais adequada para o Rio Grande do Sul e nordeste da Argentina, em função da inclinação solar. Essa orientação faz que ocorra sombreamento na maior parte do dia na entrelinha do plantio.

O manejo de plantas daninhas, após o transplante das mudas, foi realizado em duas etapas: a primeira ( $1^{\circ}$ ano), quatro semanas após o transplante (set. 1999), foi aplicada à mistura de haloxyfop-R-methyl (Mirage), na dose de $0,36 \mathrm{~kg} \mathrm{ha}^{-1}\left(1,50 \mathrm{~L} \mathrm{ha}^{-1}\right.$ ), com atrazine (Agar Cross Atrazina) a $1,50 \mathrm{~kg} \mathrm{ha}^{-1}\left(3,0 \mathrm{~L} \mathrm{ha}^{-1}\right)$ acrescido de Dowfax (adjuvante), na concentração de 0,5\% v/ v. A segunda aplicação foi realizada aos 90 dias após o transplante (nov. 1999), entre os camalhões (fileiras de Pinus taeda), utilizando-se a mistura de glyphosate (Roundup) a 1,44 kg ha-1 (3,0 $\left.\mathrm{L} \mathrm{ha}^{-1}\right)$, com atrazine (Agar Cross Atrazina) a 2,0 kg ha-1 $\left(4,0 \mathrm{~L} \mathrm{ha}^{-1}\right)$, acrescidos de Dowfax (adjuvante), na concentração de 0,5\% v/v e com as mudas de Pinus taeda protegidas.

O segundo período de aplicação dos herbicidas (aplicação de $2^{\circ}$ ano) foi realizado em março e setembro de 2000. Foi aplicada, entre os camalhões de Pinus taeda, a mistura de glyphosate (Roundup) a 1,44 kg ha $^{-1}$ (3,0 L ha-1), com atrazine (Agar Cross Atrazine) a $1,50 \mathrm{~kg} \mathrm{ha}^{-1}\left(3,0 \mathrm{~L} \mathrm{ha}^{-1}\right)$, acrescidas de Dowfax (adjuvante) na concentração de $0,5 \% \mathrm{v} / \mathrm{v}$.

Foram realizadas avaliações nas 10 plantas centrais do camalhão central, quanto a: sobrevivência (\%), altura (cm) da planta, diâmetro do colo $(\mathrm{cm})$ da planta e o fator produtividade $\left(\mathrm{cm}^{3}\right)$, aos 12 e 24 meses após o transplante das mudas.

A sobrevivência das plantas de Pinus taeda foi determinada através de porcentagem de plantas vivas. Os valores da sobrevivência de plantas foram transformados em arco-seno, para fins de normalização de sua distribuição. Para isso, foi usado o pacote estatístico ESTAT 2.0 UNESP - FCAV - Jaboticabal, SP.

Para a altura, as plantas foram medidas desde a base da planta no solo até a gema apical, sendo o diâmetro medido com um paquímetro junto ao colo da muda e o fator de produtividade, determinado pela fórmula:

Fator produtividade $\left(\mathrm{cm}^{3}\right)=\mathrm{DC}^{2} . \mathrm{H} / 1000$

em que:

$$
\begin{aligned}
& \mathrm{DC}=\text { diâmetro do colo }(\mathrm{cm}) ; \mathrm{e} \\
& \mathrm{H}=\text { altura da planta }(\mathrm{cm}) .
\end{aligned}
$$

Para determinar o acúmulo de biomassa das plantas daninhas, foram amostradas duas áreas de $0,25 \mathrm{~m}^{2}$ no camalhão central, entre a $3^{\mathrm{a}} / 4^{\mathrm{a}}$ e $9^{\mathrm{a}} / 10^{\mathrm{a}}$ mudas de Pinus taeda, no primeiro ano. Já no segundo ano as plantas daninhas foram coletadas entre a $4^{\mathrm{a}} / 5^{\mathrm{a}}$ e a $8^{\mathrm{a}} / 9^{\mathrm{a}}$ muda. Após o corte rente ao solo, as plantas foram secadas em estufa durante 72 horas à temperatura de $65^{\circ} \mathrm{C}$. O material foi pesado e os resultados, convertidos em kg.ha-1 .

As principais plantas daninhas que ocorreram na área foram Axonopus compressus (Poaceae), Paspalum sp. (Poaceae), Andropogon lateralis (Poaceae), Cyperus sp. (Cyperaceae), Conyza bonariensis (Asteraceae), Gnaphallium purpureum (Asteraceae), Oxalis sp. (Oxalidaceae), Schizachirium microstachyum (Poaceae), Elyonorus muticuns (Poaceae), Vernonia chamaedrys (Asteraceae), Eryngium eberneum (Apiaceae), Wedelia padulosa (Asteraceae) e outras espécies que não foram identificadas. De acordo com Montenegro (1999), a porcentagem de espécies encontradas no campo San Miguel é de 63\% de gramíneas, 30\% dicotiledôneas e $7 \%$ de ciperáceas.

\section{RESULTADOS E DISCUSSÕES}

A análise dos dados de sobrevivência (\%) não revelaram diferenças significativas (Teste $F$ ) entre os tratamentos aplicados, conforme se pode visualizar nas Tabelas 3 e 4. 
Tabela 3 - Resultados da análise de variância de sobrevivência (\%), desenvolvimento em altura (cm), diâmetro do colo (mm) e fator de produtividade $\left(\mathrm{cm}^{3}\right)$ de Pinus taeda

Table 3 - Analysis of variance of survival rate (\%), height development $(\mathrm{cm})$, stem diameter $(\mathrm{mm})$ and yield $\left(\mathrm{cm}^{3}\right)$ of Pinus taeda

\begin{tabular}{|c|c|c|c|c|c|}
\hline$\overline{F V}$ & G.L. & $\begin{array}{c}\text { Sobrev. }(\%) \\
\text { Q.M. }\end{array}$ & $\begin{array}{c}\text { Alt. (cm) } \\
\text { Q.M. }\end{array}$ & $\begin{array}{c}\mathrm{DC}(\mathrm{mm}) \\
\text { Q.M. }\end{array}$ & $\begin{array}{c}\text { FP }\left(\mathrm{cm}^{3}\right) \\
\text { Q.M. }\end{array}$ \\
\hline Blocos & 2 & $34,9 \mathrm{NS}$ & $3096,8 *$ & $102,7 \mathrm{NS}$ & $222655,2 *$ \\
\hline Tratamentos & 4 & $132,8 \mathrm{NS}$ & $3689,6 * *$ & $715,6 * *$ & $843230,9 * *$ \\
\hline Resíduo & 8 & 87,1 & 365,4 & 38,4 & 43382,1 \\
\hline TOTAL & 14 & & & & \\
\hline
\end{tabular}

Sendo FV: Fonte de variação; GL: graus de liberdade; QM: quadrado médio; NS: não-significativo; * diferença significativa (5\%) e ** diferença significativa $(1 \%)$.

Tabela 4 - Resultados do teste de Tukey dos valores de sobrevivência (\%), desenvolvimento em altura (cm), diâmetro do colo $(\mathrm{cm})$ e fator de produtividade de Pinus taeda

Table 4 - Tukey's test for survival rate (\%), height development (cm), stem diameter $(\mathrm{cm})$ and yield $\left(\mathrm{cm}^{3}\right)$ of Pinus taeda

\begin{tabular}{|c|c|c|c|c|}
\hline Tratamento & $\begin{array}{c}\text { Sobrevivência } \\
(\%)\end{array}$ & $\begin{array}{l}\text { Altura } \\
(\mathrm{cm})\end{array}$ & $\begin{array}{l}\text { Diâmetro do } \\
\text { Colo }(\mathrm{cm})\end{array}$ & $\begin{array}{c}\text { Fator de } \\
\text { Produtividade }\left(\mathrm{cm}^{3}\right)\end{array}$ \\
\hline Testemunha & $74,8 \mathrm{a}^{*}$ & $153,9 \quad b$ & $3,78 \quad b$ & $2,20 \quad \mathrm{c}$ \\
\hline Controle camalhão 1 ano & 83,4 a & 228,1 a & $6,30 \mathrm{a}$ & $9,05 \quad b$ \\
\hline Controle camalhão 2 anos & 89,3 a & 244,2 a & 7,69 a & 14,44 a \\
\hline Controle total 1 ano & 89,3 a & 224,5 a & $6,29 \mathrm{a}$ & $8,88 \quad b$ \\
\hline Controle total 2 anos & $77,5 \mathrm{a}$ & $223,0 \mathrm{a}$ & 7,43 a & $12,31 \mathrm{a} \mathrm{b}$ \\
\hline Média & 82,8 & 214,8 & 6,30 & 9,38 \\
\hline $\mathrm{C} \mathrm{V} \%$ & 11,3 & 8,9 & 9,8 & 17,8 \\
\hline
\end{tabular}

* Médias de tratamentos não seguidas por mesma letra, dentro de cada variável, diferem entre si, pelo teste de Tukey a 5\% de probabilidade de erro.

Esse resultado pode ser explicado pela proteção das mudas durante a aplicação química; na testemunha, em que a infestação poderia influenciar a sobrevivência, o efeito da construção dos camalhões reduziu a quantidade de plantas daninhas próximas às mudas de Pinus taeda, logo após o plantio.

Existem evidências em vários estudos em relação ao manejo da vegetação competidora sobre o crescimento das mudas de espécies florestais. Na maioria dos casos, o manejo da vegetação nativa produz aumento na sobrevivência e crescimento das árvores (WHITE et al., 1990; PERRY et al., 1993; SOUTH et al., 1993).

Apesar de os resultados não apresentarem diferença significativa entre as intensidades de controle, ou seja, um período ou dois períodos, para as variáveis altura e diâmetro do colo, para a variável fator de produtividade, com o controle sendo feito no camalhão, a diferença foi significativa. Kogan e Figueroa (1999) concluíram que, ao não controlar plantas daninhas no segundo período de crescimento em Pinus radiata, perderamse $65 \%$ de incremento em biomassa $\left(D^{2} \mathrm{H}\right)$.

Na Figura 1 são apresentados dados relativos ao desenvolvimento inicial do Pinus taeda nas variáveis ou características avaliadas diante das modalidades e intensidades de controle.

Pezzutti (2000), em estudo realizado na região do nordeste argentino, citou, com relação a para Pinus taeda, que o controle nas primeiras etapas do plantio é de grande importância. Aos 3 anos de idade das plantas, o referido autor não encontrou diferença significativa entre tratamentos com controles realizados durante um, dois e três períodos de crescimento, mas todos eles diferiram significativamente da testemunha, sem controle. Esse mesmo autor ainda citou Yeiser (1999), que obteve um acréscimo de produtividade do Pinus taeda em $26,9 \%$ para altura (cm) e $65,2 \%$ para diâmetro de altura do colo $(\mathrm{mm})$ em relação à testemunha, em trabalhos com controle de plantas daninhas nos dois primeiros anos de plantios.

R. Árvore, Viçosa-MG, v.30, n.5, p.711-718, 2006 
(A)

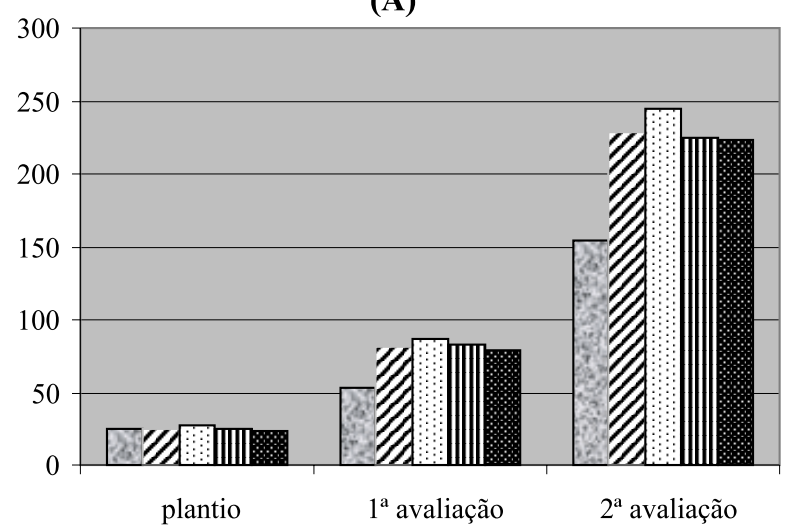

(B)

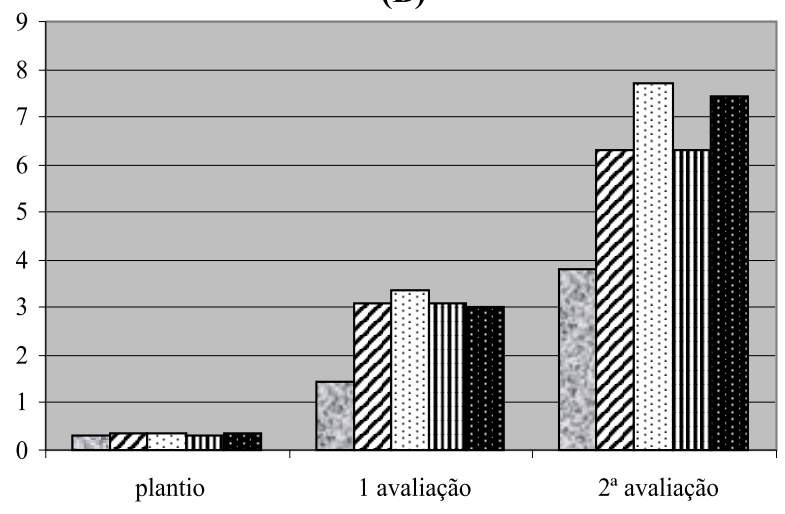

(C)

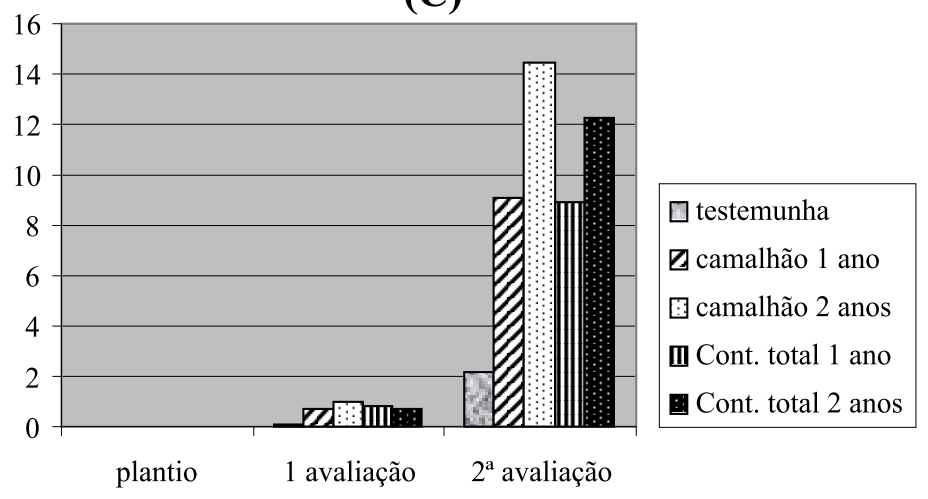

Figura 1 - Desenvolvimento inicial do Pinus taeda das variáveis altura em cm (A), diâmetro do colo em $\mathrm{cm}$ (B) e fator de produtividade $\mathrm{em}^{3}(\mathrm{C})$.

Figure 1 - Pinus taeda initial development of variables height in $\mathrm{cm}(A)$, stem diameter in $\mathrm{cm}(B)$ and yield in $\mathrm{cm}^{3}(C)$.

Kogan e Figueroa (1999), realizando estudos experimentais no Chile, ao controlar plantas daninhas descreveram que a intensidade de controle, durante os dois primeiros anos, em Pinus radiata, limitou-se à linha do plantio, não sendo necessário o controle em superfície total.

De acordo com estudo efetuado por Montenegro (1998), houve um acréscimo de 60\% em diâmetro do colo $(\mathrm{mm})$ após um ano de controle, em relação à testemunha. No entanto, Jokela et al. (2000) descreveram um ganho de $43 \%$ em relação à testemunha após um controle de cinco anos para essa mesma espécie. Em estudo realizado por Cañellas et al. (1999) sobre comportamento de Pinus pinea, fertilização e aplicação de herbicidas em Mayorga - Espanha, concluiu-se que o emprego de herbicidas incrementou o crescimento longitudinal das plantas do gênero Pinus mais que com os fertilizantes.

Pelos resultados do presente estudo apresentados na Tabela 5, quanto ao desenvolvimento inicial em altura e diâmetro do colo, o melhor tratamento em relação a testemunha apresentou um incremento de 58,7 e 103,4\% para o Pinus taeda, respectivamente.

Quanto ao volume ou fator de produtividade, o melhor tratamento apresentou um incremento de 557,0\% em comparação com a testemunha. No entanto, quando avaliados os resultados por um período maior na rotação, Maclaren (1993), em plantios de Pinus radiata, descreveu que aos 11 anos de idade apresentou uma diferença de $50 \%$ de desenvolvimento em volume entre árvores que cresceram com controle de plantas daninhas e as que cresceram sem esse controle. 
Tabela 5 - Porcentual de acréscimo nas características altura, diâmetro do colo (DC) e fator de produtividade (FP) em decorrência dos tratamentos aplicados no plantio de Pinus taeda, em relação à testemunha sem controle. Santa Maria, RS

Table 5 - Percent increase in height, stem diameter (SD) and yield as a function of treatments applied to Pinus taeda planting compared with the control. Santa Maria, RS

\begin{tabular}{lccc}
\hline & Altura $(\mathrm{cm})$ & DC $(\mathrm{mm})$ & $\mathrm{FP}\left(\mathrm{cm}^{3}\right)$ \\
\cline { 2 - 4 } Camalhão 1 ano & $48,2 \%$ & $66,7 \%$ & $311,8 \%$ \\
Camalhão 2 anos & $58,7 \%$ & $103,4 \%$ & $557,0 \%$ \\
Total 1 ano & $45,9 \%$ & $66,4 \%$ & $304,1 \%$ \\
Total 2 anos & $44,9 \%$ & $96,6 \%$ & $460,1 \%$ \\
\hline
\end{tabular}

Fox (2000), avaliando o efeito da competição de herbáceas e arbustos em plantios de Pinus taeda, nos Estados Unidos, analisados após oito anos, descreveu que não houve diferença significativa de um ou dois períodos de controle, com um volume de $40 \mathrm{~m}^{3} \mathrm{ha}^{-1} \mathrm{e}$ $38 \mathrm{~m}^{3} \mathrm{ha}^{-1}$, respectivamente. Porém, quando comparada com a testemunha sem controle, a diferença foi bastante significativa, apresentando um volume de $14 \mathrm{~m}^{3} \mathrm{ha}^{-1}$.

Estudos avaliados no decorrer de 13 anos em plantios de Pinus taeda e Pinus echinata, aplicando controle de vegetação herbácea e arbustiva por cinco anos, comprovaram que a diferença no desenvolvimento inicial segue pela rotação, aumentando no decorrer dos anos, atingindo, no final dos 13 anos, o tratamento com controle total, a um volume de $285 \mathrm{~m}^{3} \mathrm{ha}^{-1}$, enquanto a testemunha apresentou um volume de $165 \mathrm{~m}^{3} \mathrm{ha}^{-1}$ (CAIN, 1999). O mesmo autor concluiu, ainda, que o resultado de ganho em volume $\left(\mathrm{m}^{3} \mathrm{ha}^{-1}\right)$ do melhor tratamento eqüivaleu a três anos de crescimento da testemunha.

A massa seca aparente apresentada na Tabela 6 indicou que o manejo foi eficiente, utilizando-se mistura de diferentes herbicidas. Moyano et al. (2004) relataram que Haloxyfop-p-metil e atrazina aplicados em Pinus taeda apresentam um controle mais prolongado, diminuindo quantidades de aplicações e custos. Panizza et al. (2004), em plantios de Pinus taeda no Uruguai, concluíram que a mescla de Haloxyfop-p-metil + Sulfometuron apresentou o maior controle de ciperáceas por parte do Sulfometuron e de gramíneas por parte do Haloxyfop-p-metil.

Na Tabela 6, pode-se visualizar que o manejo reduziu significativamente a quantidade de plantas daninhas, tendo, assim, correlação negativa entre biomassa de plantas daninhas e volume do Pinus taeda, ou seja, quanto maior um, menor o outro.

Mesmo que esses resultados citados anteriormente sejam provenientes de áreas, climas, solos e composição vegetal diferentes, apontaram que a maior influência da competição no Pinus taeda se dá nos primeiros anos, devendo continuar a avaliação no tempo que poderá resultar em melhor tratamento, em relação custobenefício, bem como manejo somente na linha e por um período.

Tabela 6 - Quantificação da massa seca aparente (kg.ha- $\left.{ }^{-1}\right)$ das espécies de plantas daninhas coletadas no camalhão da área de estudo. Santa Maria, RS

Table 6-Measurement of apparent dry mass $\left(\mathrm{kg}_{\mathrm{h}} \mathrm{ha}^{-1}\right)$ of weed species collected at the ridge in the study area. Santa Maria, $R S$

\begin{tabular}{lcc}
\hline Tratamentos & $1^{\circ}$ ano & $2^{\circ}$ ano \\
\hline Testemunha & 2180,3 & 5409,2 \\
Controle camalhão 1 ano & 612,6 & 2739,0 \\
Controle camalhão 2 anos & 532,8 & 483,8 \\
Controle total 1 ano & 648,3 & 2352,4 \\
Controle total 2 anos & 560,4 & 600,2 \\
\hline
\end{tabular}

\section{CONCLUSÕES}

De acordo com o estabelecimento, condução e avaliação desta pesquisa, os resultados permitiram concluir que:

a) A sobrevivência das mudas de Pinus taeda não foi afetada pela modalidade e intensidade de manejo.

b) Quanto à modalidade de manejo, ou seja, controle total ou controle no camalhão, não houve diferenças para a espécie de Pinus taeda.

c) Para a intensidade de manejo, um ano ou dois anos, o melhor tratamento foi o manejo por dois períodos.

\section{REFERÊNCIAS BIBLIOGRÁFICAS}

CAIN, M. D. Woody and herbaceous competition effects on stand dynamics and growth of 13year-old natural, precommercially thinned loblolly and shortleaf pines. Canadian Journal Forest Research, v. 29, p. 947-959, 1999.

CAÑELLAS, I. et al. Comportamiento de planta de Pinus pinea en vivero y campo: ensayos de tecnicas de cultivo de planta, fertilizacion y aplicacion de herbicidas. Revista

Investigacion Agraria, v. 8, n. 2, 1999. 
ESTAT 2.0, UNESP - FCAV - Programa de análise estatística. Jaboticabal: 1992.

FOX, R. T. Desarrollo de regímenes silviculturales, sitios específicos para Pinus taeda en la planicie costera atlántica de los Estados Unidos. In: SILVOARGENTINA, 1., 2000, Gobernador Virassoro. Anais... Gobernador Vinassoro: 2000. p. 11-47.

JOKELA, E.J.;WILSON, D.S.;ALLEN, J.E., Early growth responses of slash and loblolly Pine following fertilization and herbaceous weed control treatments at estabablishment. Southern Journal Applied Forest, v. 24, n. 1, p. 23-30, 2000.

KOGAN, M.; FIGUEROA, R. Interferencias producidas por las malezas durante los dos primeros años de establecimento de Pinus radiata D. Don, In: IUFRO CONFERENCE SILVOTECNA, "Mejoramiento productividad de sítios", 10., 1998. Concepción. IUFRO... Concepción: 1998. p.233-245.

Interferencia producida por las malezas durante los dos primeros años en Pinus radiata D. Don. Revista Bosque, v.20. n.1, p. 57-64. 1999. Acesso em 17/12/2001. Disponível em: http.//www.uach.cl/extens/revbosque/v20n1/ bq-20-1-057-064.htm.

MACLAREN, P. Radiata pine grower's manual. Chemical weed control. New Zealand, 1993. p.34-39. (FRI N.184)

MONTENEGRO, P.A., Efecto del periodo y cobertura del control de malezas en el desarrolo de plantaciones de Pinus taeda L. 1998. 110 f. (Trabalho de Conclusão da Graduação) - Universidad del Salvador, 1998.

MONTEnegro, P.A. Determinación de la associacón vegetal antes de la aplicación de herbicidas en el campo. San Miguel: Empresa La Papelera del Plata, 1999. 6 p.

MOYANO, L.; ALLIERI, L.; BONINO F. Evaluación del control postemergente de malezas com aplicaciones selectivas de haloxyfop, diclosulam y atrazina sobre plantaciones de pino en el noreste de la provincia de Corrientes, Argentina. In: CONGRESSOBRASILEIRODA CIÊNCIADAS PLANTAS DANINHAS, 24., 2004, São Pedro da Aldeia. Anais... São Pedro da Aldeia: 2004. CD-Rom.
PANIZZA, C.; BONINO F.; ALLIERI, L.

Evaluación del control de malezas post emergente de distintas mezclas de herbicidas aplicados en post plantación de pino taeda (Pinus taeda). In: XXIV CONGRESSOBRASILEIRODA CIÊNCIADAS PLANTAS DANINHAS, 24., 2004, São Pedro da Aldeia. Anais... São Pedro da Aldeia: 2004. CD-Rom.

PERRY, M.A. et al. Competitive responses of loblolly Pine, sweetgum, and broomsedge densities.

Canadian Journal of Forest Research, v. 23, p. 2049-2058, 1993.

PEZZUTTI, R., Efecto del control de malezas en el crecimiento inicial de plantaciones de Pinus taeda L. no NE de Corrientes, Argentina.

SILVOARGENTINA 1, 2000, Gobernador Virasoro, Corrientes, Argentina: 2000.

PITELLI, R.A.; MARCHI, S.R. Interferência de plantas daninhas nas áreas de reflorestamento. In. SEMINÁRIO TÉCNICO SOBRE PLANTAS DANINHAS E O USO DE HERBICIDAS EM REFLORESTAMENTO, 1., 1998, Rio de Janeiro. Anais..., Rio de Janeiro: Universidade Federal Rural do Rio de Janeiro, 1998. p. 44-64.

SAGPyA FORESTAL. Março de 1997. (Publicación de la Secretária de Agricultura, Ganaderia, Pesca y Alimentación de Argentina, n. 2).

SILVA, J. L. S. Produtividade de componentes de um sistema silvipastoril constituído por Eucalyptus saligna Smith. e pastagens cultivada e nativa no Rio Grande do Sul. 1998. 179 f. Tese (Doutorando em Zootecnia)- Universidade Federal de Viçosa, Viçosa, MG, 1998.

SOUTH, D.B.; ZWOLONSKI, J.B.; DONALD, D.G.M Interactions among seedling diameter grade, weed control, and soil cultivation for Pinus radiata en South Africa. Canadian Journal of Forest Research, v. 23, p. 2078-2082, 1993.

TEDESCO, M.J. et al. Análises de solo, plantas e outros materiais. 2. ed. Porto Alegre: Universidade Federal do Rio Grande do Sul, 1995. 174p. (Boletim Técnico de Solos, 5).

VETTORI, L. Métodos de análises do solo. Rio de Janeiro: MA/EPFS, 1969. 642p. (Boletim, 7).

WHITE, D.E.; WITHERSPOON, L.; NEWTON, M. Herbaceous weed control in conifer plantations with hexazinone and nitrogen formulations. New Forest, v. 4, p. 97-105, 1990. 